\title{
IMPACT BIOMECHANICS OF BRAIN INJURIES: A PROPOSAL FOR EVALUATING VULNERABILITY BASED ON RELIABILITY ANALYSIS
}

\author{
Máté Hazay, Imre Bojtár \\ Budapest University of Technology and Economics, Faculty of Civil Engineering \\ matehazay@hotmail.com \\ DOI: 10.17489/biohun/2017/1/08
}

\begin{abstract}
Traumatic brain injuries (TBIs) contribute to a high degree of mortality and morbidity in society. From engineering point of view, the human brain can be considered as a mechanical system which is subjected to extreme effects, since every type of TBIs are derived from the large magnitude of mechanical loads. Impact biomechanics deals with the prevention of injuries via the suitable design of safety systems. It requires the quantification of limit values of the mechanical effects that humans can tolerate. The goal of this paper is twofold. Firstly, it contains a brief literature review where some of the most important milestones and major conclusions of previous researches are mentioned. Secondly, it presents a proposal about the applicability of reliability analysis to assess the vulnerability of the human brain.
\end{abstract}

Keywords: traumatic brain injuries, vulnerability of the human brain, reliability analysis, injury risk curves

\section{Introduction}

Injury biomechanics covers several areas of study involving different parts of the human body. Among these fields the investigation of traumatic brain injuries (TBIs) can be considered as a special area due to the epidemiological importance of TBIs. TBIs contribute to a high degree of mortality and morbidity in society, and motor vehicle accidents are the leading source of blunt impact-induced head injuries. ${ }^{1,2}$ As it was reported in the middle of the 1990s, approximately 1,7 million TBIs occurred in the United States every year. ${ }^{3}$

The main aim of impact biomechanics is the prevention of injuries via the suitable design of passive restraint systems. This procedure requires the quantification of limit values of the mechanical effects that humans can tolerate. Intensive research work has been carried out for decades to determine such tolerance levels, however, this work is far from finished yet. The human brain can be considered as a mechanical system which is subjected to mechanical loads which could cause injuries. These loads can be considered as extreme effects in a sense that they have large magnitude (comparing to those loads which arise every day), relatively rare occurrence and extreme consequences (brain injury). The determination of tolerance values can be considered as a fragility analysis where those effects are quantified which would cause the failure of the mechanical system.

Early researches have led to the development of several head injury predictors which could be adopted into motor vehicle safety standards around the world. Indeed, due to the development of safety systems in cars over the last 20 years, the number of TBIs have been reduced, however there are indications that this trend is slowing down and TBIs still represent major diseases wordwide. One possible reason for that is the roughness of existing injury criteria. Nowadays the evaluation of passive safety 
system of cars is based on the Head Injury Criteria (HIC), which was originally proposed by Versace ${ }^{5}$ at the beginning of the 1970 s. HIC takes into account the integral of the linear acceleration pulse $a(t)$, measured at the centre of gravity of the head. The evaluation is performed in a way that a HIC value is calculated according to Equation 1:

$$
H I C=\left[\frac{1}{t_{2}-t_{1}} \int_{t_{1}}^{t_{2}} a(t) \mathrm{d} t\right]^{2,5}\left(t_{2}-t_{1}\right),
$$

where $t_{1}$ and $t_{2}$ are arbitrary initial and final times during the acceleration pulse, are chosen to maximize $H I C$, and this value is compared to a limit value which is prescribed in the particular standard. Although the evaluation methodology of restraint systems based on HIC has been used for more than forty years, several doubts exist corresponding to the applicability of the currently used procedure which indicates the necessity of further research. In the next section a brief literature review is presented where some of the most important milestones and conclusions of previous researches are shown. These conclusions help understand the potential weaknesses of the currently used safety standards which initiated new research methodologies, including the determination of tissue-level tolerances with finite element analyses based on accident reconstructions and experiments performed on living cell cultures. Afterwards a proposal is made about the applicability of reliability analysis which may be an appropriate tool in the future for performing a detailed vulnerability analysis of the human brain.

\section{Previous methods and main results}

At the beginning, impact biomechanical researches started with experimental investigations on cadavers, animals or certain type of physical models. The main goals were to iden- tify and describe injury mechanisms and to determine tolerance limits. In these researches the occurrence of injury was connected to a certain value of so-called input parameters which are characteristics of the external mechanical load which acts on the human head. Among the first efforts the skull fracture experiments of Wayne State University should be mentioned which have led to the development of the first tolerance curve ${ }^{6}$ with regards to the occurrence of head injury. Furthermore, researches have been performed where the injury mechanisms of different types of brain injuries have been analyzed. After experiments, contusions have been associated with the increase of intracranial pressure due to the relative motion between the skull and the brain and the local bending of the skull. ${ }^{78}$ Contusion may occur not just at the site of the impact (coup) but at areas distal to the loading site (contrecoup) ${ }^{9,10}$ (Figure 1).

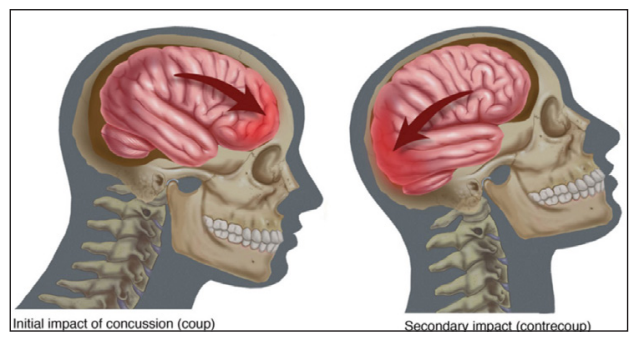

Figure 1. Coup and contrecoup injuries ${ }^{11}$

Later, contusion was identified as a possible outcome of the movements of the brain against rough and irregular skull surfaces. ${ }^{12}$ Subdural and epidural hematoma can also occur due to the relative motion between the brain and the skull which causes shear strains, elongation and the rupture of bridging veins. ${ }^{13}$ After the vein rupture, neural tissue is compressed due to the increased pressure which can cause cell death. Injury mechanisms of intracerebral hematoma (Figure 2) are similar to those which have been mentioned for contusions. ${ }^{14,15}$ Furthermore, since different pressure values arise at the coup and contrecoup locations, a pressure gradient exists which creates shear stress- 
es in neural tissue which can also cause focal injuries. ${ }^{16,17}$

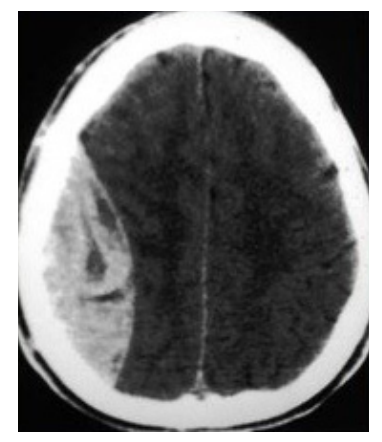

Figure 2. Epidural hematoma ${ }^{18}$

Diffuse injuries form a spectrum of injuries ranging from mild concussion to diffuse axonal injury (DAI). Axonal damage is thought to occur due to shear strains and stresses (Figure 3) which occurs in the neural tissue during the trauma. ${ }^{19}$ However, by now it is known that the axonal damage does not necessarily occur at the moment of the trauma, but the shear effects cause an axolemmal permeability dysfunction which can lead to axonal damage through different phenomena. ${ }^{20}$

After the identification of injury mechanism, injury thresholds should be quantified. In or- der to achieve that it is important to determine which kind of external effects can cause the previously described injury mechanisms. In early studies much attention was paid on the separate investigation of the effect of linear (translational) and rotational (angular) accelerations. It has led to a debate about which effect has the more important contribution to brain injury. Holbourn ${ }^{22}$ hypothesized that rotational acceleration has a greater effect than linear accelerations in the occurrence of injury and others supported this theory. ${ }^{23}$ Two concepts existed how rotations cause injury: (i) inability of the brain to rotate within the skull causes injurious focal point shear strains and (ii) diffuse shearing of brain tissue normally located at zones of change in density. Further studies confirmed that rotational acceleration could induce severe brain injuries like concussion, ${ }^{9}$ DAI or acute subdural hematoma. ${ }^{24}$ However other researchers ${ }^{25}$ who put emphasize on the analysis of the effect of skull deformations and intracranial pressure gradients, emphasized the importance of translational accelerations in the development of head injury. As a conclusion it can be stated that both linear and rotational accelerations can cause brain injuries, and both effects have a specific role because they produce different injury mechanisms. ${ }^{26}$

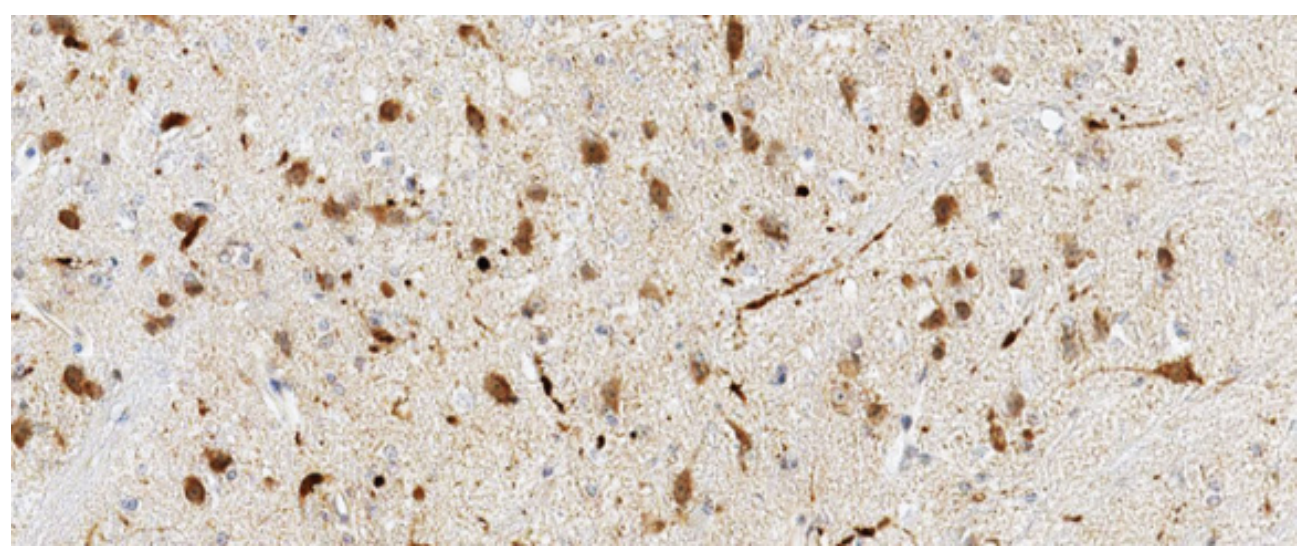

Figure 3. Axonal damage $\mathrm{e}^{21}$ 
Now it is thought that axonal injury derived from axonal stretch due to rotational acceleration, while translational acceleration is responsible for intracranial pressure induced strains and contusions.

After the recognition of the complexity of injury mechanisms and that brain injuries are outcomes of the combination of translational and rotational accelerations several doubts arose with regard to the applicability of the currently used injury metrics. One of the biggest shortcoming of HIC is that it takes into account solely the linear accelerations, ${ }^{27}$ however previously it has been shown that rotational accelerations have an important contribution to brain injuries. Furthermore, HIC is a so-called input variable based injury criterion, because it is based on linear accelerations which are the characteristics of the external load. However, it has been questioned whether it is enough to use a metric which takes into account only the external effects and does not consider the strains and stresses which arise in the highly deformable components of the brain. ${ }^{28} \mathrm{Ad}$ ditionally, the believed contribution of HIC values to the failure probability is questionable. The currently used limits of HIC values are derived from the risk curves of Prasad and Mertz, ${ }^{29}$ which were determined based on cadaver experiments. However, in these experiments only skull fracture and hematoma creation due to artery rupture were analyzed, and other types of brain injuries were not considered. Despite these doubts, HIC is widely used to evaluate the performance of passive restraint systems. In the past several changes have occurred in the safety standards where the acceptable HIC value and the time interval where the integral (in Equation 1) is calculated have been altered, but the main concept of HIC has remained unchanged. One major reason for that is its easy applicability. Such an input variable based metric has the advantage that the evaluation of crash tests is simple, since the acceleration pulse is easily obtained due to the sensors placed on the dummies.

The above mentioned doubts motivated have researchers to continue the investigations in order to establish more robust injury metrics. A new trend has developed where $3 D$ finite element head models are used to perform numerical experiments. This approach allows us to work with relatively precise material parameters and geometry, while both linear and rotational accelerations can be considered. These kinematic variables serve as an input data of these simulations where so-called output (i.e. dependent) variables like stresses, strains and pressures are calculated. This possibility initiated a tremendous research effort in order to identify so-called tissue-level tolerances (i.e. threshold values of stresses, strains and pressures).

By now, many different models exist in the literature which differ in certain geometrical characteristics, material parameters and boundary conditions. Usually these numerical models are validated against cadaver experimental data. ${ }^{30,31}$ One of the most widely used models is the University College of Dublin Brain Trauma Model (UCDBTM) (Figure 4) of Horgan and Gilchrist ${ }^{32,33}$ which is freely available at BEL Repository. ${ }^{34}$

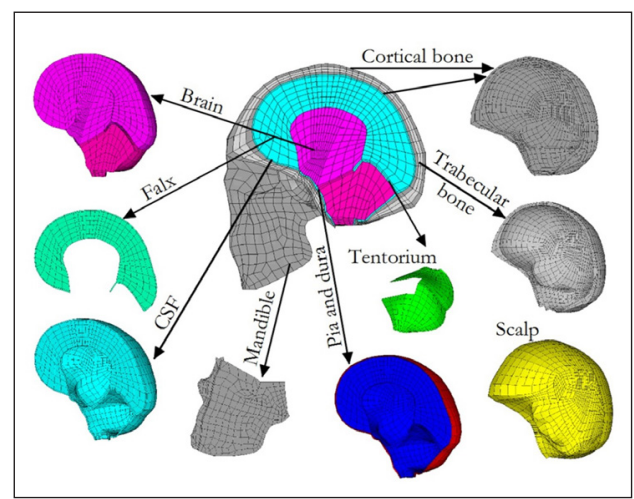

Figure 4. The University College of Dublin Brain Trauma Model 
Researches for tissue-level tolerances usually involve accident reconstructions. ${ }^{35-37}$ Usually dynamic simulations are performed to calculate those kinematic variables which acted on the head and later these loads are placed to the finite element head model to calculate the output variables. Since the clinical outcome of these accidents are known, the calculated stresses, strains and pressure values can be used to estimate human tissue-level tolerances. As a result, several proposed threshold values were published, ${ }^{38,39}$ however these results have a large deviation which can be attributed partly to the natural variability among humans, uncertainty in the reconstruction process and modelling uncertainty due to the application of the particular finite element model and imperfect failure criteria. Due to the large deviation of results, it is not reasonable to adjust one specific tolerance value, therefore such a conclusion was made that future investigations should be based on a probabilistic basis where the possibility of brain injury is determined. The latest trend is to draw risk curves where a distribution is used to describe injury tolerance limit. ${ }^{39-42}$ These curves express conditional probability, since they show that how large the injury probability is if the characteristics of the effect (which can be either an input or an output variable) has a specific intensity.

\section{Discussion and proposal}

The previous literature overview intends to outline three major conclusions:

- In order to perform a more reliable vulnerability analysis of the human brain, injury description should be based on tissue-level variables (i.e. stresses, strains, pressures) instead of kinematic variables.

- The vulnerability of the brain is affected by several parameters which have a large variability. It indicates that instead of a deterministic approach with the ap- plication of one specific threshold value, tolerance assessment should be based on a stochastic approach where the probability of head injury is determined in terms of risk curves.

- Although the applicability of HIC is questionable, only some details have been changed in the previous decades while the main idea has remained unaltered. It suggests that even if further researches follow a tissue-level approach, the resulting criterion should be expressed in terms of input variables, because it would be easier applicable to evaluate passive restraint systems in the engineering practice

By now different techniques were recommended to determine risk curves, and in many impact biomechanical studies these curves have been developed for different body regions, injury types and severity classes. ${ }^{41}$ These techniques usually involve a regression analysis, where some kind of distribution function (e.g. normal, log-normal, Weibull or log-logistic) is fitted to sample points which were obtained from experiments. Although it is questionable, that such an analysis where patient specific kinematic loads were placed to the same head model is capable of describing the effect of major kinds of uncertainties which can affect the shape of the resulting risk curve. For example, since the same head model is used the effect of the natural variability of geometrical and material parameters among humans is neglected. Due to this variation, instead of a deterministic modelling approach, a probabilistic approach should be applied where a spectrum of output variables are determined. ${ }^{43}$ Moreover, if the same finite element head model is used, then it is not possible to take into account the modelling uncertainty which may have an important effect on the shape of the risk curves, since several open questions exist corresponding to the biomechanical modelling of human brain. ${ }^{43}$ Furthermore, in case of a risk curve where 
injury probability is expressed in terms of an input variable, calculations should include that uncertainty which derives from the variability of the loading curve shape.

As a matter of fact, in case of the vulnerability analysis of mechanical systems exposed to extreme effects, there is a long tradition of expressing the fragility of the system in terms of fragility curves, where the probability of failure is shown in the function of intensity of the applied load. Injury risk curves mentioned above correspond to these fragility curves where the vulnerability of the human brain is analyzed. It is well known that the shape (e.g. the slope) of these curves highly affected by the amount of uncertainties which derive from several sources. In other engineering fields (e.g. earthquake engineering, nuclear engineering) the so-called full probabilistic approach is applied in many cases ${ }^{44,45}$ to consider the effect of different types of uncertainties during the calculation. This is accomplished via defining random variables whose standard deviation describe the variability of the considered parameter. In most cases these random variables can be characterized approximately which implies that the calculated failure probability is only an estimation, still the full probabilistic approach can be considered as a state-of-art technique to characterize the fragility of a mechanical system. With the application of random variables failure probability can be calculated by reliability analysis ${ }^{46,47}$ instead of the statistical evaluation of a limited number of sample points. During the application of reliability analysis different failure modes (e.g. injury types) can be considered trough the definition of failure components where each component is characterized by a limit-state function. A limit-state function separates the sample space into a safety and a failure domain. Theoretically the failure probability can be calculated as the integral of the joint probability density function of the random variables over the failure domain.
However, instead of evaluating these integral analytically, usually numerical techniques are applied to calculate the probability of failure. The theoretical background of the different techniques and further details of the reliability analysis can be found elsewhere. ${ }^{46,47}$

Our proposal is based on the idea that the previously mentioned full probabilistic methodology could be used to perform a more detailed vulnerability analysis of the human brain. Following this aim, some remarks are mentioned about the framework of the reliability analysis (Figure 5) which will serve as a starting point of our future research. It has already been mentioned that different failure components (and limit-state functions) should be defined for different types of injury. Then the question may arise that how the failure of the whole system should be defined. In case of brain injury, the application of a series system would be reasonable, where the mechanical system is considered to be already failed if one of the possible types of injury occurred. Another important question is the correlation among injury types, which could be answered based on clinical experiences. One of the most important conclusion of the previous studies was that the future investigations should be based on a tissue-level approach. Therefore, limit-state functions should include output parameters like calculated stresses (or strains) and limit stresses (or strains). For example Equation 2 shows a possible limit-state function $G$ :

$$
G=\sigma_{\text {limit }}-\sigma_{\text {calculated }},
$$

where $\sigma_{\text {calculated }}$ is a stress value (effect) which was calculated from the finite element simulation and $\sigma_{\text {limit }}$ is the limit value of stresses (resistance). The value of $\sigma_{\text {limit }}$ should be a realization of a random variable $\sum_{\text {limit }}$ which describes tissue-level tolerance. In order to perform a detailed vulnerability analysis, the application of such a variable which describes the 
variability of tissue-level tolerance among humans is essential, since this variability was one of the major reasons for implementing probabilistic approach and risk curves. The characterization of these variables could be based on the results of previous studies where accident reconstructions were performed. Another important issue is to take into account the uncertainty which derives from the random shape of possible time-acceleration curves. Previous studies showed that this variability can highly influence the magnitude of brain deformation. ${ }^{48}$ Analogously to earthquake engineering, this task could be obtained by repeating finite element simulations for several time-acceleration records which have been normalized in a way that these records represent approximately the same intensity. Such time-acceleration records for crash tests can be obtained from the National Highway Traffic Safety Administration (NHTSA) database. In future studies further random variables may be applied as well. In many cases, terms in the limit-state functions are multiplied by random variables which describe modelling uncertainties. Such a variable could be characterized based on a detailed research work, where several finite element head models are used with different complexity, geometry, material parameters and boundary conditions to observe the variability of calculated results deriving from our modelling approach. By scaling time-acceleration records, the reliability analysis can be repeated for several times for different intensity (e.g. HIC) values in order to obtain discrete points (injury probabilities for given intensity values) on which distribution function can be fitted to obtain the injury risk curve.

Probably the application of the full probabilistic approach will produce many questions corresponding to the characterization and the number of the applied random variables, selection and normalization of time-history records, definition of limit-state functions, etc. However, this approach has the advantage that every type of uncertainties can be described by random variables, and there is a possibility that the most important variables can be characterized approximately based on results of previous and

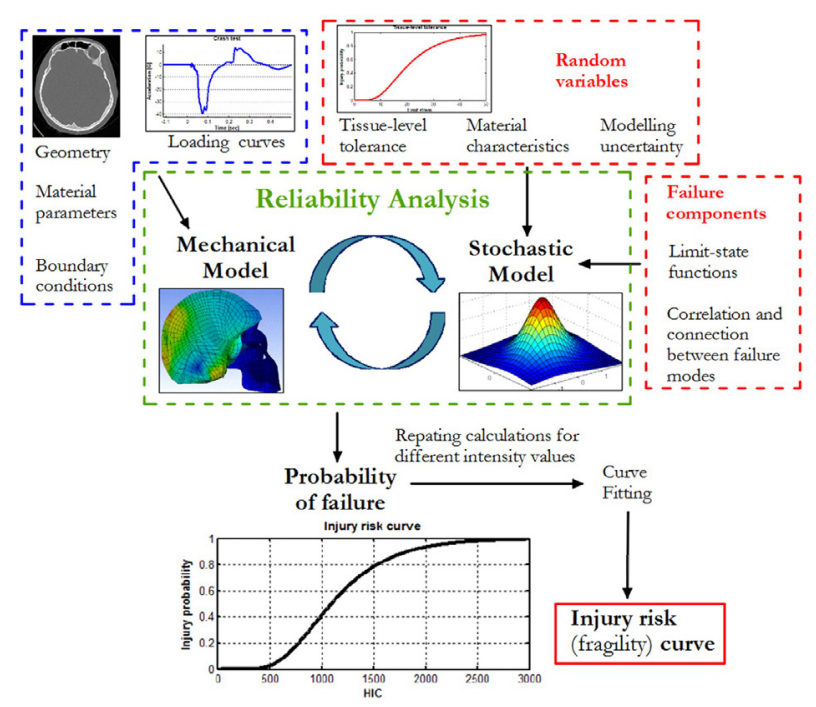

Figure 5. Framework of the reliability analysis 
current researches. Furthermore, the proposed methodology fulfils those requirements that injury evaluation is based on a tissue-level variables, while injury risk curves are expressed in terms of input variables (e.g. HIC values). Obviously, there is a lot of work to do in order to determine reliable risk curves for brain injuries and reliability analysis may become one of the state-of-art techniques in the future whose application will lead to the improvement of restraint systems and occupant safety.

\section{REFERENCES}

1. B King AI. Introduction to and applications of injury biomechanics. In: Yoganandan N, Nahum $A M$, Melvin JW, editors. Accidental injury: biomechanics and prevention. New York: Springer Science+Business Media; 2015. p. 1-31.

2. Yoganandan N, Pintar FA, Zhang J, Gennarelli $T A$, Beuse N. Biomechanical aspects of blunt and penetrating head injuries. In: Gilchrist $M D$, editor. IUTAM proceedings on impact biomechanics: from fundamental insights to applications. Dordrecht: Springer;2005.p.173-84.

3. Sosin DM, Sniezek JE, Thurman DJ. Incidence of mild and moderate brain injury in the United States, 1991. Brain Injury 1996;10(1):47-54.

4. Kleiven S. Predictors for traumatic brain injuries evaluated through accident reconstruction. Stapp Car Crash Journal 2007;51:81-114.

5. Versace J. A Review of the Severity Index. In proceedings of the 15th Stapp Car Crash Conference. SAE Technical Paper 710881, 1971 DOI: $10.4271 / 710881$.

6. Lissner HR, Lebow M, Evans FG. Experimental studies on the relation between acceleration and intracranial pressure changes in man. Surg Gynecol Obstet 1960;111:329-38.

7. Courville CB. The mechanism of coup-contrecoup injuries of the brain: A critical review of recent experimental studies in the light of clinical observations. From: Bulleting of the Los Angeles Neurological Society 1950 Jun; 15(2):72-86.

8. Gurdjian ES, Gurdjian ES. Re-evaluation of the biomechanics of blunt impact injury of the head. Surg Gynecol Obstet, 1975 Jun; 140(6):845-50.

9. Ommaya AK, Grubb RL Jr, Neumann RA. Coup and contre-coup injury: observations on the mechanics of visible brain injuries in the rhesus monkey. J Neurosurg 1971; Nov; 35(5):503-16.
10. Bandak FA, Eppinger RH. A three-dimensional finite element analysis of the human brain under combined rotational and translational accelerations. Proceedings 38th Stapp Car Crash Conference; 1994 Oct 31 - Nov 2; Fort Lauderdale, FL, SAE Technical Paper 942215, 1994, DOI: $10.4271 / 942215$.

11. Sport Performance Institute. Retrieved August 15, 2016, Available from: URL: http://www.spiutica.com/blog-article-concussion-in-sports.

12. Viano DC, King AI, Melvin JW, Weber K. Injury biomechanics research: an essential element in the prevention of trauma. Journal of Biomechanics 1989; 22(5):403-17.

13. Kleiven $S$. Influence of impact direction on to the human head in prediction of subdural hematoma. Journal of Neurotrauma, 2003 Apr; 20(4):365-79.

14. Gurdjian ES, Gurdjian ES. Acute head injury: A review. Surgery Annual 1980;12:223-41.

15. Gennarelli TA, Thibault LE. Biomechanics of acute subdural hematoma. Journal of Trauma 1982 Aug; 22(8):680-6.

16. Thomas LM, Roberts VL, Gurdjian ES. Impact-induced pressure gradients along three orthogonal axes in the human skull. Journal of Neurosurgery 1967 Mar; 26(3):316-21.

17. Ommaya AK, Gennarelli TA. Cerebral concussion and traumatic unconsciousness: Correlation of experimental and clinical observations on blunt head injuries. Brain 1974 Dec; 97(4): 633-54.

18. Wikipedia, The Free Encyclopedia. (n. d.) Retrieved September 19, 2017, Available from: URL: https://en.wikipedia.org/wiki/Epidural_hematoma. 
19. Strich SJ. Shearing of nerve fibers as a cause for brain damage due to head injury. The Lancet 1961 Aug; 278(7200):443-8.

20. Poulishock JT, Buki A, Koiziumi H, Stone J, Okonkwo DO. Initiating mechanisms involved in the pathobiology of traumatically induced axonal injury and interventions targeted at blunting their progression. Acta Neurochir Suppl 1999; 73:15-20.

21. Wikipedia, The Free Encyclopedia. (n. d.) Retrieved September 19, 2017, Available from: URL: https://en.wikipedia.org/wiki/Diffuse_axonal_injury.

22. Holbourn AHS. Mechanics of head injuries. The Lancet 1943; 2:(6267):438-41.

23. Gurdjian ES, Hodgson VR, Thomas LM, Patrick $L M$. Significance of relative movements of scalp, skull, and intracranial contents during impact injury to the head. Journal of Neurosurgery 1968 Jul; 29(1):70-2.

24. Gennarelli TA, Abel JM, Adams H, Graham D. Differential tolerance of frontal and temporal lobes to contusion induced by rotational acceleration. In Proceedings of the 23rd Stapp Car Crash Conference, 1979 Oct 17-19, San Diego, CA. SAE Technical Paper 791022, 1979, DOI: $104271 / 791022$.

25. Gurdjian ES, Lissner HR, Latimer FR, Haddad $B F$, Webster JE. Quantitative determination of acceleration and intracranial pressure in experimental head injury; preliminary report. Neurology 1953 Jun; 3(6):417-23.

26. Hardy WN, Foster CD, Mason MJ, Yang KH, King AI, Tashman S. Investigation of head injury mechanisms using neutral density technology and high-speed biplanar X-ray. Stapp Car Crash Journal 2001 Nov; 45:337-68.

27. Goldsmith W. Current controversies in the stipulation of head injury criteria. Journal of Biomechanics 1981; 14(12):883-4.

28. King AI, Yang KH, Zhang L, Hardy W, Viano $D C$. Is head injury caused by linear or rotational acceleration? In Proceedings of the IRCOBI Conference, 2003 Sep 24-27, Lisbon, Portugal, 2003:1-12.

29. Prasad P, Mertz HJ. The position of the United States Delegation to the ISO working group 6 on the use of $\mathrm{HIC}$ in the automotive environment.
SAE paper No. 851246, 1985.

30. Nahum AM, Smith $R$, Ward CC. Intracranial pressure dynamics during head impact. Proceedings 21st Stapp Car Crash Conference, 1977 Oct 19-21, SAE Technical Paper 770922, 1977, DOI: $104271 / 770922$.

31. Trosseille X, Tarriére C, Lavaste F, Guillon F, Domont $A$. Development of a FE.M. of the human head according to a specific test protocol. Proceedings of the 36th Stapp Car Crash Conference - 1992 Nov 2-4, Seattle, WA SAE Technical Paper 922527, 1992, DOI:104271/922527.

32. Horgan TJ, Gilchrist MD. The creation of three-dimensional finite element models for simulating head impact biomechanics. International Journal Crash 2003 Jan; 8(4):353-66.

33. Horgan TJ, Gilchrist MD. Influence of FE model variability in predicting brain motion and intracranial pressure changes in head impact simulations. International Journal Crash 2004 Aug; 9(4):401-18.

34. Biomechanics European Laboratory. Available from: URL: http://www.tecno.ior.it/VRLAB/ through the BEL Repository managed by Istituti Ortopedici, Rizzoli, Bologna, Italy, 2004.

35. Doorly MC, Horgan TJ, Gilchrist MD. Reconstruction of Head Injury Cases Arising from Falls Using the UCD Brain Trauma Model. In: Gilchrist MD, editor. IUTAM Proceedings on Impact Biomechanics: From Fundamental Insights to Applications. Dordrecht: Springer, 2005: p. 431-40.

36. Viano DC, Casson IR, Pellman EJ, Zhang L, King $A I$, Yang $K H$. Concussion in professional football: Brain responses by finite element analysis: Part 9. Neurosurgery 2005;57(5):891-916.

37. Kleiven $S$. Predictors for traumatic brain injuries evaluated through accident reconstruction. Stapp Car Crash Journal 2007 Oct;51:81-114.

38. Takhounts EG, Eppinger RH, Campbell JQ, Tannous RE, Power ED, Shook LS. On the development of the SIMon Finite Element Head Model, 47th Stapp Car Crash Journal 2003;47:107-33.

39. Viano DC. Head Impact Biomechanics in Sport. In: Gilchrist MD, editor. IUTAM Proceedings on Impact Biomechanics: From Fundamental Insights to Applications. Dordrecht: Springer; 2005. p. 121-30. 
40. Mertz HJ, Irwin AL. Anthropomorphic test devices and injury risk assessments. In: Yoganandan N, Nahum AM, Melvin JW, editors. Accidental injury: biomechanics and prevention. New York: Springer Science+Business Media, 2015:83-112.

41. Petitjean A, Trosseille X, Yoganandan N, Pintar $F A$. Normalization and scaling for human response corridors and developmennt of injury risk curves. In: Yoganandan N, Nahum AM, Melvin $J W$, editors. Accidental injury: biomechanics and prevention. New York: Springer Science+Business Media, 2015:769-92.

42. Prasad $P$. Injury criteria and motor vehicle regulations. In: Yoganandan N, Nahum AM, Melvin $J W$, editors. Accidental injury: biomechanics and prevention. New York: Springer Science+Business Media, 2015. p. 793-810.

43. Yang KH, King AI. Modeling of the brain for injury simulation and prevention. In: Miller $K$, editor. Biomechanics of the brain. New York: Springer Science+Business Media, 2011. p. 91110.

44. Shinozuka M, Hwang H, Reich M. Reliability assessment of reinforced concrete containment structures. Nuclear Engineering and Design 1984;80:247-67.

45. Pinto PE, Franchin P, Lupoi A, Lupoi G. Seismic fragility analysis of structural systems. Journal of Engineering Mechanics 2006;132(4):385-95.

46. Nowak AS, Collins KR. Reliability of structures. New York: McGraw-Hill, 2000.

47. Lemaire $M$. Structural reliability. London: ISTE Ltd and Hoboken: John Wiley \& Sons, 2009.

48. Post A, Hoshizaki TB, Gilchrist MD. Finite element analysis of the effect of loading curve shape on brain injury predictors. Journal of Biomechanics, 2012 Feb; 45:679-83.

\section{Máté Hazay}

Budapest University of Technology and Economics, Faculty of Civil Engineering H-1111 Budapest, Műegyetem rkp. 3.

Tel.: (+36) 1 463-1434 